\title{
Weekly forecast of the Settlement Price of Differences in the Brazilian short-term market through recurrent artificial neural networks
}

\author{
Fabiane Barbosa do Nascimento, Leonardo Rocha Olivi, Luís Henrique Lopes Lima, \\ Leonardo Willer de Oliveira, Ivo Chaves da Silva Junior \\ Departamento de Energia Elétrica, Universidade Federal de Juiz de Fora, \\ Juiz de Fora-Minas Gerais (e-mail: fabiane.barbosa@engenharia.ufjf.br).
}

\begin{abstract}
The Settlement Price of Differences is the short-term price published weekly by the Electric Energy Trading Chamber, which is a relevant variable for the free electricity market. Monitoring predicting its behavior allows market participants to be able to define their strategies assertively, resulting in successful energy contracting with the right amounts at the right time and at lower prices. These prices are influenced by several factors, mainly related to uncertainties of demand and hydrology, resulting in their excessive variability as dynamic systems. The present work proposes the use of Recurrent Artificial Neural Networks in order to assist the decision process of the purchase of energy in the Free Energy Market. The artificial neural network of the proposed model is trained through the resilient backpropagation algorithm and is applied to the Brazilian energy market. The network showed high performance, being able to perform a weekly prediction, with results presented up to 24 weeks ahead, for the Southeast / Center-West submarket, with a relevant hit level, facilitating the decision making for short term market agents.
\end{abstract}

Resumo: O Preço de Liquidação das Diferenças é o preço de curto prazo divulgado semanalmente pela Câmara de Comercialização de Energia Elétrica, sendo uma variável relevante para o mercado livre de energia elétrica. Acompanhá-lo e prever seu comportamento permite que os participantes do mercado consigam definir suas estratégias de maneira assertiva, resultando em contratações energéticas bemsucedidas, com os montantes adequados, no momento certo e com menores preços. Estes preços são influenciados por diversos fatores, principalmente associados a incertezas sobre demanda e hidrologia, resultando em sua excessiva variabilidade como sistemas dinâmicos. O presente trabalho propõe a utilização de Redes Neurais Artificiais Recorrentes visando auxiliar o processo de decisão da compra de energia no Mercado Livre de Energia. A rede neural artificial do modelo proposto é treinada por meio do algoritmo resilient backpropagation e é aplicada ao mercado brasileiro de energia. A rede mostrou desempenho elevado, sendo capaz de realizar uma predição semanal, com resultados apresentados de até 24 semanas à frente, para o submercado Sudeste/Centro-Oeste, com nível de acerto relevante, facilitando a tomada de decisão para agentes do mercado de curto prazo.

Keywords: Artificial Neural Networks, Settlement Price of Differences, Free Energy Market, Energy Price Prediction, Stochastic Systems.

Palavras-chaves: Redes Neurais Artificiais, Preço de Liquidação das Diferenças, Mercado Livre de Energia, Predição de Preço de Energia, Sistemas Estocásticos.

\section{INTRODUÇÃO}

A energia elétrica é um fator determinante para que um país cresça e desenvolva seu comércio e indústria, de modo a criar oportunidades para seus cidadãos. Para algumas empresas, a energia ocupa um papel tão essencial quanto ao da sua própria matéria-prima, tecnologia ou mão-de-obra. Desta forma, a energia elétrica é um assunto fundamental para uma política de desenvolvimento e para estratégias de mercado, e a garantia de sua disponibilidade é imperativa.

A comercialização de energia elétrica no Brasil está segmentada em dois ambientes de contratação: i) o Ambiente de Contratação Regulada (ACR), em que se realizam as operações de compra e venda entre agentes vendedores e de distribuição, mediante contrato jurídico, ressalvados os casos previstos em lei, conforme regras e procedimentos de comercialização específicos; ii) Ambiente de Contratação Livre (ACL), em que se realizam as operações de compra e venda de energia elétrica, objeto de contratos bilaterais livremente negociados, conforme regras e procedimentos de comercialização específicos (Brasil, 2004).

No caso ACL, para os clientes que consomem uma quantidade maior que a previamente contratada, é possível realizar contratos no mercado de curto prazo, em que as diferenças contratuais são valoradas por meio do Preço de Liquidação das Diferenças (PLD) (ABRACEEL, 2019), que é publicado semanalmente pela Câmara de Comercialização de Energia Elétrica (CCEE, 2019).

O cálculo do PLD está intrinsicamente conectado com as características operativas do setor elétrico brasileiro, predominantemente hidrelétrico. Os recursos e fatores 
hídricos têm características sazonais e variações de natureza probabilística, o que atribui ao PLD uma natureza estocástica, com grande variabilidade temporal e volatilidade em suas tendências (Sousa, 2013).

O presente trabalho aborda o tema descrito por meio de modelos de séries temporais baseados em Redes Neurais Artificiais (RNA) Recorrentes. O modelo de RNA empregado é do tipo Time Delay Neural Network (TDNN) e o trabalho proposto apresenta resultados baseados na entrada de dados históricos do PLD. A Seção 2 apresenta os trabalhos correlatos, a Seção 3 apresenta a metodologia proposta, a Seção 4 apresenta os resultados e, finalmente, a Seção 5 apresenta as conclusões.

\section{TRABALHOS CORRELATOS}

A predição do Preço de Liquidação das Diferenças é importante para os consumidores que contratam seu fornecimento no Mercado Livre de Energia. Conhecer os valores comportamentais do PLD auxiliam a definir estratégias de contratação e emprego eficiente da energia, estabelecendo assim, contratos que visam minimizar os riscos para as empresas (Garcia, 2011). A natureza probabilística do PLD leva a um problema de modelagem para predição de resultados futuros. Diversas abordagens são relatadas na literatura, dentre as quais destacam-se a teoria dos jogos (Azevedo, 2004), modelos fundamentais e modelos de séries temporais (Queiroz et al., 2007; Molina et al., 2011; Reston Filho et al., 2014). Os autores de Molina et al. (2011) apresentaram em seu trabalho um método que permite obter os preços de liquidação do mercado chileno.

Para os modelos baseados em séries temporais, os autores de Queiroz et al. (2007) utilizaram RNA para tratar dados relativos a preços spot de energia elétrica no Brasil. O trabalho mostrou técnicas combinadas em seu escopo e resultados satisfatórios com foco na gestão de risco e análise de investimentos. Ainda sobre séries temporais, os autores de Reston Filho et al. (2014) combinaram técnicas para o problema de predição de preço no mercado brasileiro de energia elétrica, contando com a mescla de RNA tipo perceptron na generalização dos dados e modelos autorregressivos (ARIMA) aplicados aos dados de entradas da perceptron multicamadas.

As RNA podem aproximar universalmente funções, com capacidade de aprendizado, podendo incorporar as dinâmicas e nuances dos dados. Depois de bem calibradas, apresentam complexidade computacional reduzida, sendo computacionalmente rápidas (Silva et al., 2015). Diferentemente do outros trabalhos, as RNAs são utilizadas neste trabalho com uma aplicação voltada para sistemas dinâmicos contaminados por variáveis aleatórias por meio de autorregressão uma vez que o PLD é composto por diversas componentes aleatórias como demanda e fatores hidrológicos, dentre outros fatores estocásticos.

\section{METODOLOGIA}

Nesta seção, apresentam-se os detalhes da rede neural artificial implementada para a predição do Preço de Liquidação das Diferenças, com sua topologia e considerações para treinamento e validação.

\subsection{Topologia}

Por se tratarem de dados estocásticos e dinâmicos, a RNA utilizada é do tipo recorrente com modelo de Elman, a qual realimenta as saídas passadas como novas entradas para o sistema de aprendizado, adquirindo comportamento autorregressivo, melhor adaptada para lidar com o problema em questão. Nesta abordagem os resultados produzidos pelos neurônios de saída são realimentados às suas entradas por meio de atrasos temporais. A Figura 1 mostra a topologia da rede utilizada, bem como as entradas escolhidas para gerar, como saída, a predição do próximo valor futuro do PLD.

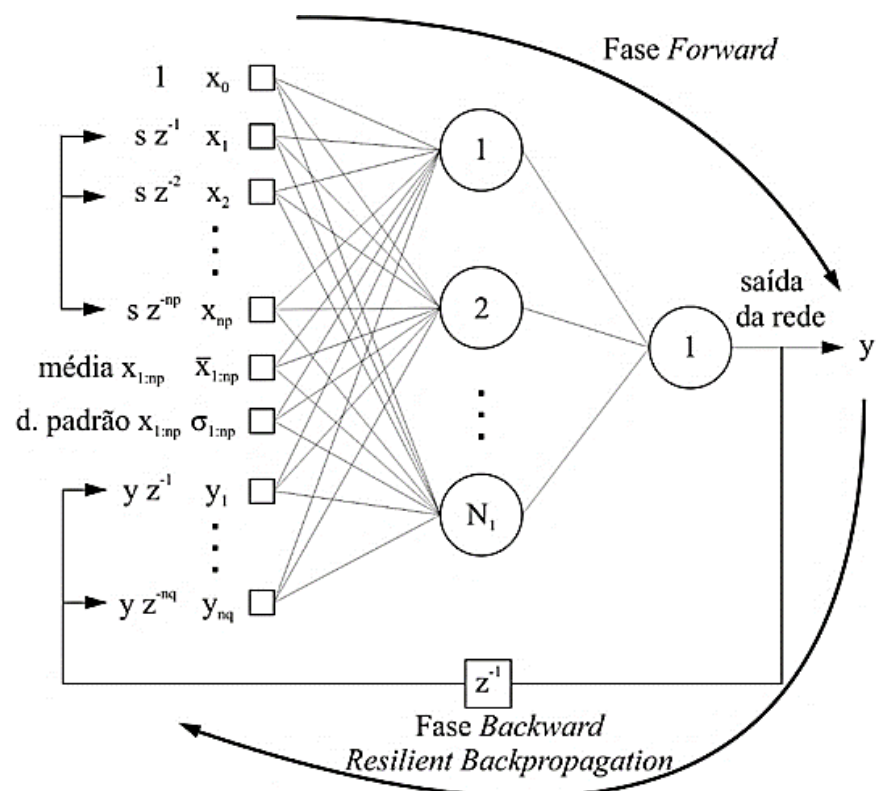

Figura 1 - Rede neural artificial recorrente utilizada.

Desta maneira, conforme mostra a Figura 1, a rede neural empregada possui as seguintes entradas: a série temporal do PLD com $n_{p}$ entradas atrasadas, gerando uma estrutura tipo TDNN; $\mathrm{n}_{\mathrm{q}}$ entradas atrasadas em TDNN referentes às próprias saídas da rede, inserindo a recorrência $\mathrm{e}$ comportamento autorregressivo, fator desejado para sistemas dinâmicos estocásticos; e, mais duas entradas de estatísticas referentes aos dados, a média e o desvio padrão das $n_{p}$ entradas atrasadas no tempo atual, inserindo assim as tendências estatísticas dos dados na predição.

O PLD é formado por diversas variáveis explanatórias, como Energia Armazenada, Geração Hidráulica, Carga de Energia, dentre outras, disponibilizadas pelo Operador Nacional do Sistema (ONS, 2019; Reston Filho et al., 2014), que poderiam servir de entradas para a Rede Neural. No entanto, optou-se por utilizar somente o PLD, evitando assim a necessidade de predição das variáveis explanatória a fim de usar esses dados na predição do PLD, pois estas possuem elementos aleatórios, sendo, portanto, o mesmo problema fundamental do PLD. Assim, evita-se a acumulação de erros em cascata nas entradas.

As redes neurais artificiais podem contar com diversas camadas de neurônios, as quais podem ser interpretadas geometricamente como a composição sucessiva de regiões. De acordo com o Teorema da Aproximação Universal (Haykin, 2001; Silva et al., 2015), quando uma rede neural é 
utilizada para aproximar de funções, uma única camada intermediária de neurônios se faz necessária, e por essa razão, esta é a estrutura apresentada na Figura 1.

A base de dados utilizada no presente trabalho foi obtida na Câmara de Comercialização de Energia Elétrica (CCEE, 2019). Os dados abrangem o período de 21/03/2015 à 08/09/2017, totalizando 132 semanas, do PLD médio referente ao submercado Sudeste/Centro-Oeste. Embora os resultados apresentados neste trabalho sejam obtidos para a região Sudeste/Centro-Oeste, quaisquer outras regiões poderiam ser utilizadas uma vez que seus comportamentos são similares.

Este trabalho optou por utilizar dados de aproximadamente 2 anos (112 semanas) em razão do PLD ter seus valores máximos e mínimos recalculados anualmente, bem como o sistema que o compõe estar em constante atualização. Assim, a utilização de dados de um longo período de tempo inseriria as tendências de um sistema desatualizado nas predições, levando a uma possível fonte de inconsistências.

$\mathrm{Na}$ arquitetura escolhida, os neurônios artificiais possuem funções de ativação tangente hiperbólicas, que saturam no intervalo [-1,1]. Dessa maneira, os dados do PLD devem ser normalizados na faixa dinâmica da função de ativação. Uma vez normalizados os dados estão aptos a serem aplicados na rede, por meio da fase forward, também descrita na Figura 1. A normalização é feita por meio de relação linear, apenas transpondo os limites das variáveis para o intervalo aceito pela função de ativação, sem a descaracterização dos dados.

A etapa forward, para a rede desenvolvida, é descrita por:

$y=\tanh \left(\beta\left(\omega_{2}\left(\tanh \left(\beta\left(\omega_{1} x+\theta_{1} x_{0}\right)\right)\right)+\theta_{2} x_{0}\right)\right)$

em que $\omega_{1}, \theta_{1}, \omega_{2}, \theta_{2}$ são os pesos sinápticos das camadas escondida e de saída, respectivamente; a função de ativação dos neurônios da camada escondida e de saída é a tangente hiperbólica e $\beta$ é o seu fator de inclinação da parte dinâmica. Como os neurônios da camada de saída não são lineares, é necessário que os dados sejam desnormalizados pelo processo inverso ao utilizado na normalização aplicada ao PLD, sendo esta apenas de uma operação de reescala.

\subsection{Treinamento e validação}

Para o treinamento, dividiu-se o conjunto de dados normalizados, sendo os 88 primeiros para treinamento $(78,57 \%)$ e os 24 últimos para validação $(21,43 \%)$, uma vez que se deseja prever, passo a passo - ou, conforme a periodicidade dos dados, semana a semana - até 24 semanas afrente do PLD, aproximadamente seis meses no futuro. O conjunto de treinamento é responsável pelo aprendizado da rede e ajuste dos pesos sinápticos. O segundo conjunto é utilizado para avaliar o desempenho de generalização da rede.

O método de treinamento utilizado no presente trabalho é o Resilient Backpropagation. É um método bastante eficiente, tendo convergência computacional rápida, se igualando em eficiência a métodos de segunda ordem quasi-Newton. Além disso, este método é capaz de lidar com mínimos locais durante o treinamento, sendo uma escolha interessante para o problema (Haykin, 2001; Silva et al., 2015).
Nele, iniciam-se os primeiros vetores de pesos sinápticos com valores aleatórios e assim é possível calcular as primeiras saídas da na fase forward, mostrada na Equação 1. A saída gerada é utilizada para calcular o erro quadrático médio (MSE, Mean Squared Error), por meio do qual é possível definir se a rede convergiu ou não. O MSE é definido por:

$M S E=\sum \frac{1}{2}(d-y)^{2}$

em que $d$ são os dados do PLD e $y$ é a saída da rede neural dada na Equação 1. O processo de treinamento da rede envolve a minimização do MSE por meio da fase backward, exibida na Figura 1. A fase backward do método resilient backpropagation depende dos Gradientes $\nabla$ do MSE com relação a cada um dos pesos sinápticos, dados pelo conjunto de equações a seguir:

$$
\begin{aligned}
& \nabla M S E_{\omega_{1,2}}=\frac{\partial}{\partial \omega_{1,2}}\left(\sum \frac{1}{2}(d-y)^{2}\right) \\
& \nabla M S E_{\theta_{1,2}}=\frac{\partial}{\partial \theta_{1,2}}\left(\sum \frac{1}{2}(d-y)^{2}\right)
\end{aligned}
$$

De posse dos Gradientes das Equações 3 e 4 é possível, então, regular os pesos sinápticos da rede neural de modo que estes se adaptem aos dados a serem aprendidos, reduzindo o MSE. $O$ ajuste dos pesos sinápticos $p=\{\omega, \theta\}$ é dado por:

$p^{\{t+1\}}=p^{\{t\}}-\eta_{p} \operatorname{sinal}\left(\nabla M S E_{p}\right)$

em que a taxa de aprendizado $\eta$ é uma matriz ajustada por:

$$
\eta_{p}^{\{t+1\}}(i, j)=\left\{\begin{array}{l}
\eta^{+} \eta_{p}^{\{t\}}(i, j), \text { se } \nabla M S E_{p}^{\{t-1\}} \nabla M S E_{p}^{\{t\}}>0 \\
\eta^{-} \eta_{p}^{\{t\}}(i, j), \text { se } \nabla M S E_{p}^{\{t-1\}} \nabla M S E_{p}^{\{t\}}<0 \\
\eta_{p}^{\{t\}}(i, j), \text {, se } \nabla M S E_{p}^{\{t-1\}} \nabla M S E_{p}^{\{t\}}=0
\end{array}\right.
$$

em que $p$ representa todos os pesos sinápticos existentes na rede neural, e $\eta_{p}(i, j)$ representa cada um dos pesos de ajuste para cada uma das matrizes de pesos sinápticos, dado pela Equação 6, na qual $\eta^{+}$é um incremento percentual e $\eta^{-}$um decremento percentual, tal que $0<\eta^{-}<1<\eta^{+}$. Desta maneira, a cada interação, cada um dos elementos $(i, j)$ contidos na matriz $\eta_{p}$ são ajustados individualmente.

A predição é realizada ao longo do treinamento, em que o MSE da predição também é calculado. O critério de parada é a variação do Gradiente atingir um limiar pequeno. O MSE da predição também é levado em conta como critério de parada para o treinamento. A predição sempre levará em conta as últimas entradas atrasadas no tempo, suas medidas estatísticas (média e desvio padrão), bem como as últimas saídas produzidas pela rede, conforme disposto da Figura 1.

\section{RESULTADOS}

Este capítulo apresenta os resultados obtidos por meio da aplicação da metodologia proposta nas seções anteriores para os dados reais de PLD obtidos da Câmara de Comercialização de Energia Elétrica (CCEE) e demonstra a eficácia do método utilizado com predição. Este capítulo apresentará a avaliação do treinamento bem como da predição, em duas etapas, uma de curtíssimo prazo, apenas uma semana no futuro, e a segunda prevendo várias semanas no futuro, aproximadamente 6 meses. 


\subsection{Calibração da Rede Neural Recorrente}

Para avaliar a performance de uma rede neural, utiliza-se um conjunto de dados que não está presente no processo de treinamento. Após treinar a rede, estes dados são comparados com os dados previstos pela rede, permitindo obter os erros de predição por meio parâmetros usuais encontrados na literatura, como médias, desvios padrões, intervalos de confiança e a raiz quadrada do erro quadrático médio (Root Mean Squared Error, RMSE), dado por:

$R M S E=\sqrt{\frac{1}{n} \sum_{i=1}^{n}\left|P_{i}-Q_{i}\right|^{2}}$

Diversas topologias de redes neurais foram testadas neste trabalho para encontrar uma configuração que pudesse aproximar de forma satisfatória as predições do PLD. As configurações testadas estão mostradas na Tabela 1.

Tabela 1 - Configurações de redes neurais testadas.

\begin{tabular}{|c|c|c|c|}
\cline { 2 - 4 } \multicolumn{1}{c|}{} & \multicolumn{3}{|c|}{ Configuração 1 } \\
\hline $\mathrm{n}_{\mathrm{p}}$ & 22 & 22 & 22 \\
\hline $\mathrm{n}_{\mathrm{q}}$ & 1 & 3 & 6 \\
\hline $\mathrm{N}_{1}$ & 25 & 25 & 25 \\
\hline & \multicolumn{3}{|c|}{ Configuração 2 } \\
\hline $\mathrm{n}_{\mathrm{p}}$ & 22 & 22 & 22 \\
\hline $\mathrm{n}_{\mathrm{q}}$ & 1 & 3 & 6 \\
\hline $\mathrm{N}_{1}$ & 40 & 40 & 40 \\
\hline & \multicolumn{3}{|c|}{ Configuração 3 } \\
\hline $\mathrm{n}_{\mathrm{p}}$ & 22 & 22 & 22 \\
\hline $\mathrm{n}_{\mathrm{q}}$ & 1 & 3 & 6 \\
\hline $\mathrm{N}_{1}$ & 70 & 70 & 70 \\
\hline
\end{tabular}

As configurações mostradas na Tabela 1 foram escolhidas de maneira empírica, por meio de um algoritmo de força-bruta evoluindo cada um dos parâmetros. Os valores apresentados são os resultados mais significativos. Para avaliar o desempenho de cada uma das configurações foram utilizados seis parâmetros estatísticos usuais, o RMSE, o MAE (Mean Absolute Error), o desvio-padrão do MAE e três níveis de intervalo de confiança, $90 \%, 95 \%$ e $99 \%$, com a rede neural prevendo, passo a passo, até 24 semanas afrente do PLD.

Os resultados estatísticos são apresentados nas Figuras 2, 3 e 4. Analisando as figuras, é possível observar a nãolinearidade na configuração dos parâmetros. Em outras palavras, aumentar o número de neurônios e realimentações não necessariamente melhorou os resultados. A melhor rede, para o caso tratado neste trabalho teve uma quantidade de neurônios e realimentações intermediária.

Em termos quantitativos, a rede com 40 neurônios e 3 realimentações das saídas obteve os melhores valores de RMSE e MAE, resultado possível de ser observado nas Figuras 2, 3 e 4, onde se pede atenção na observação da escala vertical, que varia em cada uma das figuras. Assim, as versões de 40 e 70 neurônios, ambas com 3 realimentações, serão consideradas nas predições.

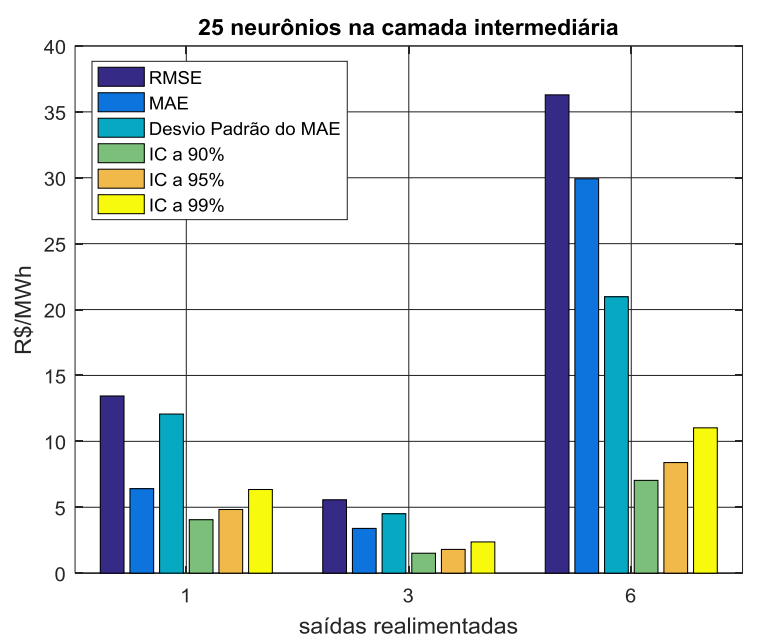

Figura 2 - Resultados da Configuração 1, para cada uma das variações no número de realimentações das saídas da rede.

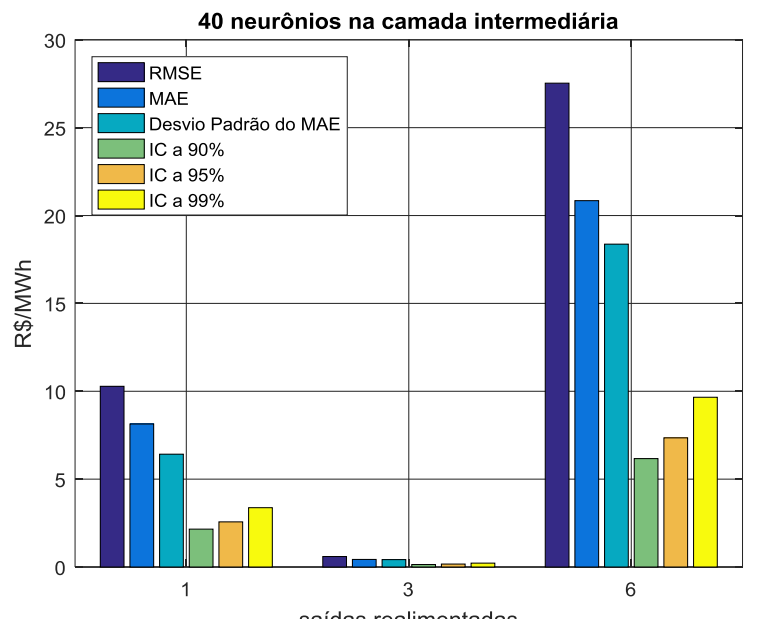

Figura 3 - Resultados da Configuração 2, para cada uma das variações no número de realimentações das saídas da rede.

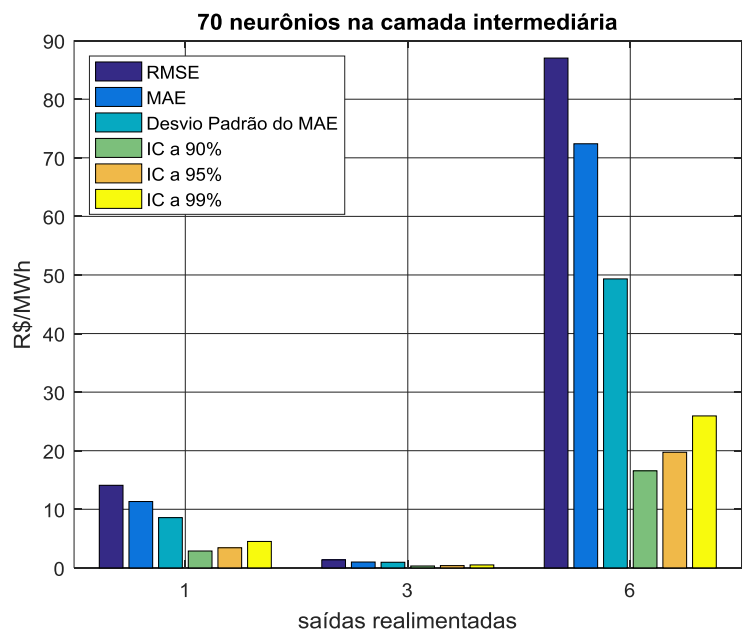

Figura 4 - Resultados da Configuração 3 para cada uma das variações no número de realimentações das saídas da rede. 


\subsection{Predição de uma semana no futuro}

A primeira abordagem de predição envolve apenas a próxima semana no futuro, de curtíssimo prazo. Assim, as 24 semanas separadas para a validação da rede neural serão utilizadas uma a uma, em sequência, e na predição da semana $i+1$ os valores das semanas anteriores $(i, i-1, \cdots)$ são conhecidos. As Figuras 5 e 6 apresentam, respectivamente, os resultados qualitativos do treinamento e da validação da rede neural com 40 neurônios na camada intermediária e 3 realimentações por meio do resilient backpropagation. A Tabela 2 indica os resultados quantitativos para a melhor rede neural obtida.

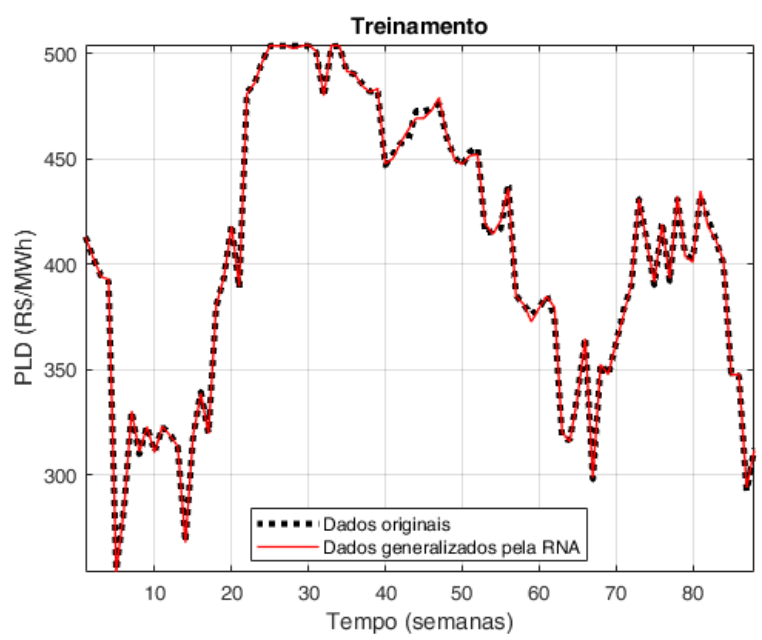

Figura 5 - Fase de treinamento para $\mathrm{N}_{1}=40$ neurônios intermediários e $\mathrm{n}_{\mathrm{q}}=3$ saídas realimentadas.

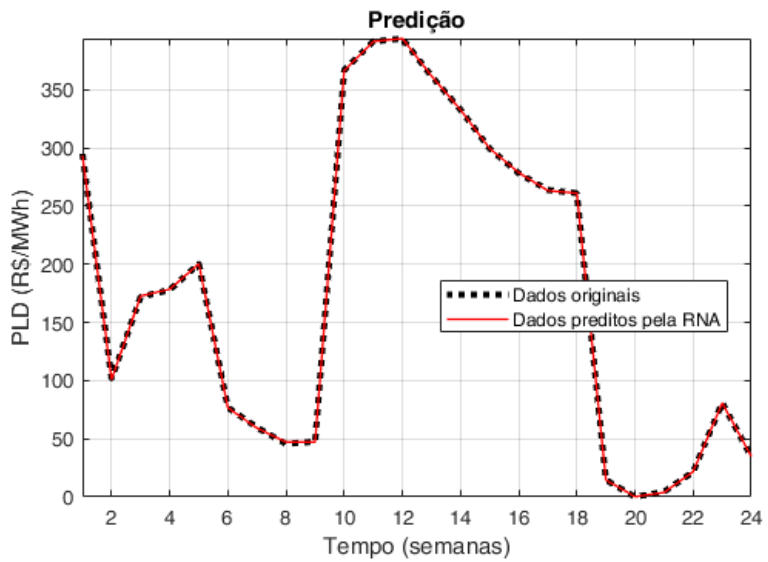

Figura 6 - Fase de validação para $\mathrm{N}_{1}=40$ neurônios intermediários e $\mathrm{n}_{\mathrm{q}}=3$ saídas realimentadas, com predição de até 24 passos no futuro, sendo um passo por vez.

Conforme é possível observar, os resultados da rede neural apresentados nas Figuras 5 e 6, bem como na Tabela 2, foram bastante satisfatórios, tanto no treinamento quanto na validação. A topologia da rede proposta foi capaz de predizer com alto nível de confiança, a cada semana, todos os dados separados para a validação da rede. Além disso, a rede também pode prever todas as tendências dos dados. Já as Figuras 7 e 8 mostram o treinamento e validação para a versão de 70 neurônios. Observa-se que a rede adquiriu características negativas de redundância no treinamento, refletindo em uma predição pobre dos dados semanais, em comparação com a versão de 40 neurônios, confirmando os dados da Seção 4.1.

Tabela 2 - Indicadores para horizontes de 4, 12 e 24 semanas com 40 neurônios e 1 passo à frente para cada rodada da rede.

\begin{tabular}{|c|c|c|c|}
\hline \multirow{2}{*}{$\begin{array}{c}\text { Indicadores } \\
\text { (R\$/MWh) }\end{array}$} & \multicolumn{3}{|c|}{ Horizonte Temporal } \\
\cline { 2 - 4 } & 4 semanas & 12 semanas & 24 semanas \\
\hline RMSE & 0,5962 & 0,7719 & 0,9783 \\
\hline MAE & 0,4311 & 0,5895 & 0,9305 \\
\hline Desvio-padrão & 0,1683 & 0,2945 & 0,3418 \\
\hline IC a 90\% & 0,1413 & 0,2471 & 0,2869 \\
\hline IC a 95\% & 0,1683 & 0,2945 & 0,3418 \\
\hline IC a 99\% & 0,2212 & 0,3870 & 0,4492 \\
\hline
\end{tabular}

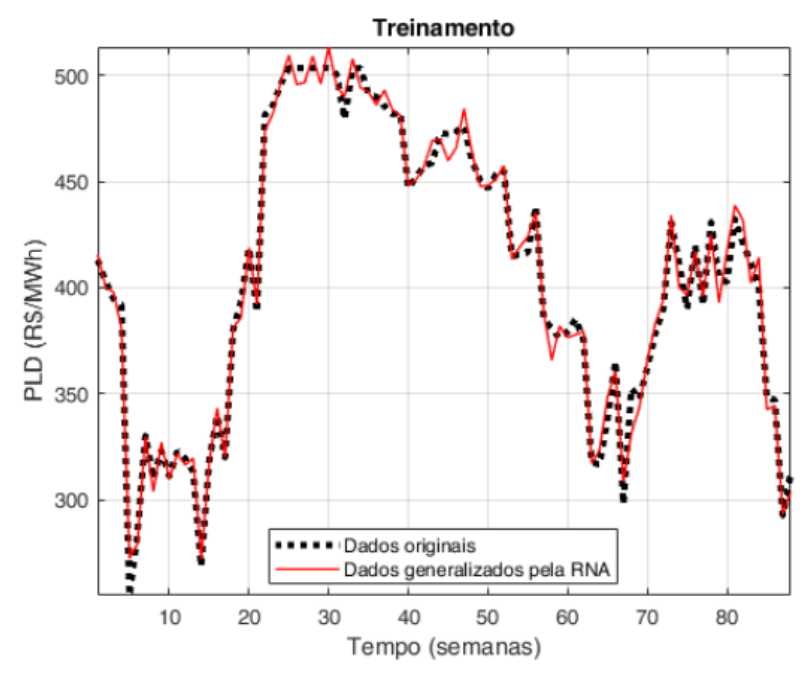

Figura 7 - Fase de treinamento para $\mathrm{N}_{1}=70$ neurônios intermediários e $\mathrm{n}_{\mathrm{q}}=3$ saídas realimentadas.

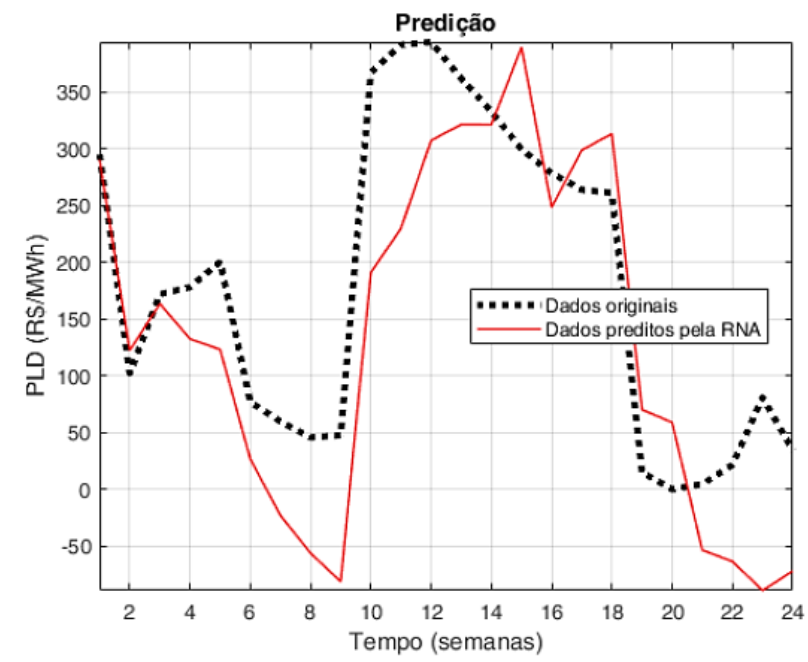

Figura 8 - Fase de validação para $\mathrm{N}_{1}=70$ neurônios intermediários e $\mathrm{n}_{\mathrm{q}}=3$ saídas realimentadas, com predição de até 24 passos no futuro, sendo um passo por vez.

\subsection{Predição de várias semanas no futuro}

Para esta seção, apresentam-se os resultados da melhor rede neural obtida, a de 40 neurônios com 3 realimentações. Nesta 
abordagem, nenhuma das 24 semanas separadas para a validação serão conhecidas pela rede neural, que utilizará suas próprias predições nas entradas referentes ao PLD.

A Figura 9 apresenta os resultados qualitativos para esta modalidade de predição. Observa-se que para a primeira semana mantém-se o comportamento obtido na modalidade da Seção 4.1, pois a primeira semana resulta do mesmo procedimento. No entanto, com o passar das semanas, os erros acumulados das predições se propagam, descaracterizando o desempenho apresentado na Figura 5. Ainda assim, observa-se que a tendência geral dos dados ainda pôde ser capturada pela abordagem, fornecendo uma ferramenta de análise a ser considerada para os especialistas da área. A Tabela 3 apresenta os dados quantitativos para o experimento, mostrando que, numericamente, o RMSE fica em torno de $\mathrm{R} \$ 6,00 / \mathrm{MWh}$.

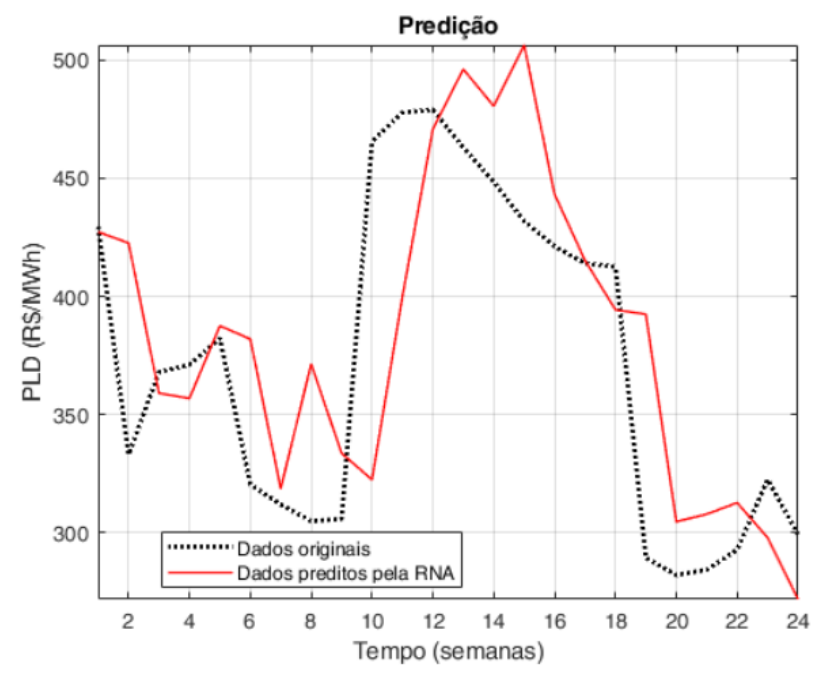

Figura 9 - Fase de validação para $\mathrm{N}_{1}=40$ neurônios intermediários e $\mathrm{n}_{\mathrm{q}}=3$ saídas realimentadas.

Tabela 3 - Indicadores para horizontes de 4, 12 e 24 semanas com 40 neurônios e 1 passo à frente para cada rodada da rede.

\begin{tabular}{|c|c|c|c|}
\hline \multirow{2}{*}{$\begin{array}{c}\text { Indicadores } \\
(\mathrm{R} \$ \text { /MWh })\end{array}$} & \multicolumn{3}{|c|}{ Horizonte Temporal } \\
\cline { 2 - 4 } & 4 semanas & 12 semanas & 24 semanas \\
\hline RMSE & 5,3645 & 6,5331 & 6,1737 \\
\hline MAE & 28,7781 & 42,6819 & 38,1144 \\
\hline Desvio-padrão & 40,8575 & 44,8616 & 36,7657 \\
\hline IC a 90\% & 67,2106 & 73,7973 & 60,4796 \\
\hline IC a 95\% & 80,0808 & 87,9287 & 72,0608 \\
\hline IC a 99\% & 105,249 & 115,5635 & 94,7084 \\
\hline
\end{tabular}

\section{CONCLUSÕES}

O presente trabalho propôs uma topologia de redes neurais artificiais para a predição semanal do Preço de Liquidação das Diferenças, a fim de prover uma ferramenta útil para analistas de dados e projetistas de tomadas de decisão acerca do mercado de curto prazo de energia elétrica brasileiro, haja vista a dificuldade de sua predição (ONS, 2019).
A rede neural proposta foi capaz de aprender corretamente os dados de treinamento, compostos por 88 semanas de operação do sistema. A predição de curtíssimo prazo, 1 semana no futuro, mostrou resultados bastante positivos para melhor configuração de rede, com nível de erro na predição abaixo de $\mathrm{R} \$ 1,00 / \mathrm{MWh}$. A mesma proposta, porém para médio prazo (6 meses), produziu um resultado satisfatório, com erro próximo a R $\$ 6,00 / \mathrm{MWh}$, e captura da tendência dos dados. Desta maneira, é possível utilizar a arquitetura proposta para também prever diferenças semanais de preços entre os submercados existentes no Brasil, sendo este também um parâmetro importante para a tomada de decisões de agências sobre a venda de energia.

\section{AGRADECIMENTOS}

Os autores agradecem o apoio do Programa de PósGraduação em Engenharia Elétrica (PPEE/UFJF), FAPEMIG, CAPES, CNPq e INERGE.

\section{REFERÊNCIAS}

ABRACEEL, Associação Brasileira Dos Comercializadores De Energia (2019). Cartilha Mercado Livre De Energia. Disponível em: <http://www.abraceel.com.br/archives/ files/abraceel_cartilha_mercadolivre_v9.pdf $>$. Acesso em: 30/04/2019.

Azevedo, E.M. (2004). Modelo Computacional De Teoria Dos Jogos Aplicado Aos Leilões Brasileiros De Energia Elétrica. Tese De Doutorado. Universidade Estadual De Campinas.

Brasil (2004), Decreto No 5.163 de 30 de Julho de 2004.

CCEE, Câmara De Comercialização De Energia Elétrica (2019). Disponível em: <http://www.ccee.org.br/>. Acesso em: 30/04/2019.

Garcia, L.H.C (2011). A Reestruturação Do Setor Elétrico Brasileiro e a Crise do Racionamento (1981-2002). Monografia. Universidade De São Paulo.

Haykin, S. (2001). Redes Neurais: Princípios E Prática. Editora Bookman.

Molina, J.P.; Zolezzi, J.M.; Contreras, J.; Rudnick, H. (2011). Nash-Cournot Equilibria In Hydrothermal Electricity Markets. Ieee Transactions On Power Systems, V. 26, N. 3, P. 1089-1101.

ONS, Operador Nacional Do Sistema (2019). Disponível em: $<$ http://www.ons.org.br/>. Acesso em: 30/04/2019.

Queiroz, A.R., Oliveira, F.A., Lima, J.W.M., Balestrassi, P.P. (2007). Simulating Electricity Spot Prices In Brazil Using Neural Network And Design Of Experiments, In: Ieee Powertech Conf. Proc., Lausanne, pp. 2029-2034.

Reston Filho, J.C.;Affonso, C.M.;Oliveira, R.C.L. (2014). Energy price prediction multi-step ahead using hybrid model in the Brazilian market. Electric Power Systems Research 117, 115-122.

Silva, I.N.; Spatti, D.H.; Flauzino, R.A. (2015). Redes Neurais Artificiais Para Engenharia e Ciências Aplicadas. Fundamentos Teóricos e Aspectos Práticos. Editora Artliber, $2^{a}$. Ed.

Sousa, A.J.S. (2013). Análise e Previsão da volatilidade do preço de liquidação das diferenças no mercado brasileiro utilizando o modelo GARCH. Dissertação de Mestrado. Universidade Federal da Bahia. 